\title{
The Power of Slicing in Internet Flow Measurement
}

\author{
Ramana Rao Kompella \\ University of California, San Diego \\ ramana@cs.ucsd.edu
}

\begin{abstract}
Network service providers use high speed flow measurement solutions in routers to track dominant applications, compute traffic matrices and to perform other such operational tasks. These solutions typically need to operate within the constraints of the three precious router resources - CPU, memory and bandwidth. Cisco's NetFlow, a widely deployed flow measurement solution, uses a configurable static sampling rate to control these resources. In this paper, we propose Flow Slices, a solution inspired from previous enhancements to NetFlow such as Smart Sampling [8], Adaptive NetFlow (ANF) [10]. Flow Slices, in contrast to NetFlow, controls the three resource bottlenecks at the router using separate "tuning knobs"; it uses packet sampling to control CPU usage, flow sampling to control memory usage and finally multi-factor smart sampling to control reporting bandwidth. The resulting solution has smaller resource requirements than current proposals (up to $80 \%$ less memory usage than ANF), enables more accurate traffic analysis results (up to $10 \%$ less error than ANF) and balances better the error in estimates of byte, packet and flow counts (flow count estimates up to 8 times more accurate than after Smart Sampling). We provide theoretical analyses of the unbiasedness and variances of the estimators based on Flow Slices and experimental comparisons with other flow measurement solutions such as ANF.
\end{abstract}

\section{Introduction}

The role of traffic measurement in operating large scale IP networks requires little or no introduction. Traffic measurement allows network operators to make informed decisions about provisioning and extending their networks, and it helps solve many operational problems. Specialized devices operating on relatively low traffic links can perform complex security analyses that reveal malicious activities [18, 20], monitor complex performance metrics [6], or simply capture packet (header) traces with accurate timestamps [7] to be analyzed offline. Much simpler solutions such as SNMP counters [16] are deployed on even the highest speed links, but they only give measurements of the total volume of the traffic. Flow level measurement at routers $[2,3]$ offers a good compromise between scalability and the complexity of the traffic analyses supported since it can offer details about the composition of the traffic mix.

In this paper, we propose a new flow measurement solution: Flow Slices. The contributions of this paper are both practical and theoretical and we summarize the most important ones here.

\author{
Cristian Estan \\ University of Wisconsin-Madison \\ estan@cs.wisc.edu
}

- Flow Slices has separate parameters controlling the three possible bottlenecks at the router: processing load, memory, and reporting bandwidth. This separation allows the solution to be applicable in a wide variety of scenarios with different resource constraints.

- The flow slicing algorithm at the core of this solution provides more accurate results than packet sampling using the same amount of memory. Moreover, it enables new measures of traffic such as estimates for the number of active flows. Note: we use Flow Slices to refer to the the complete flow measurement solution proposed in this paper and flow slicing to refer to the algorithm at the core of the solution.

- Flow Slices separates sampling rate adaptation from binning. Adaptive NetFlow uses more router memory and measurement bandwidth because its flow records are active for fixed time intervals (bins). Adaptive sampling rates give Flow Slices the robustness of Adaptive NetFlow without the overheads of binning. See Table 1 for a comparison of various flow measurement solutions.

- We propose multi-factor smart sampling that takes into account multiple factors such as byte counts, packet counts, and the existence of SYN flags in the flow records to determine the sampling probability for individual flow records. For comparable configurations, this decreases significantly the variance in estimates of the number of flow arrivals while increasing only slightly the variance for byte counts when compared to Smart Sampling.

- Optional binned measurement allows us to eliminate binning error in the analysis phase, while still maintaining the memory and reporting bandwidth overheads below those of Adaptive NetFlow.

- We propose novel estimators $\widehat{b}, \widehat{f}, \widehat{A}^{(1)}$, and $\widehat{A}^{(2)}$ for various measures of traffic. See Section 4 for a discussion of these and other estimators.

Before we explain Flow Slices, we briefly review some of the previous work in Internet flow measurement.

\section{Related work}

NetFlow [17], first implemented in Cisco routers, is the most widely used flow measurement solution today. Routers maintain flow records collecting various bits of information. Flows are identified by fields present in the header of every packet: source and destination IP address, protocol, source and destination port, and type of service bits. The flow record keeps information such as the number of 


\begin{tabular}{|l|c|c|c|}
\hline Issue & Sampled NetFlow & Adaptive NetFlow & Flow Slices \\
\hline \hline Memory usage & Variable & Fixed & Fixed \\
\hline Volume of flow data reported & Variable & Fixed & Fixed \\
\hline $\begin{array}{l}\text { Behavior under DDoS with spoofed sources } \\
\text { and other traffic mixes with many flows }\end{array}$ & $\begin{array}{c}\text { Panicky flow } \\
\text { expiration }\end{array}$ & $\begin{array}{c}\text { Reduction in } \\
\text { accuracy }\end{array}$ & $\begin{array}{c}\text { Small reduction } \\
\text { in accuracy }\end{array}$ \\
\hline Estimates of traffic in small time bins & Less accurate & Accurate & Less accurate \\
\hline Reporting overhead when using small bins & Unaffected & Large increase & Unaffected \\
\hline Lifetime of flow record in router memory & $\begin{array}{c}\text { Min (active timeout, } \\
\text { flow length }+ \\
\text { inactivity timeout) }\end{array}$ & Bin length & $\begin{array}{c}\text { Min (slice length, } \\
\text { flow length + } \\
\text { inactivity timeout) }\end{array}$ \\
\hline Resource usage at end of time bin & N/A & $\begin{array}{c}\text { Reporting spike or } \\
\text { extra memory }\end{array}$ & N/A \\
\hline Processing intensive tasks & Counting & $\begin{array}{c}\text { Counting and } \\
\text { renormalization }\end{array}$ & Counting \\
\hline Counting TCP flow arrivals (using SYNs) & Yes & $\begin{array}{c}\text { Yes } \\
\text { Separate flow } \\
\text { counting extension }\end{array}$ & Yes \\
\hline Counting all active flows & No & $\begin{array}{c}\text { Hardware flow } \\
\text { counting extension }\end{array}$ & No \\
\hline
\end{tabular}

Table 1: Sampled NetFlow, Adaptive NetFlow and Flow Slices differ in the types of measurements they support, in how they adapt to different traffic mixes, and in their resource consumption (memory usage and reporting traffic).

packets in the flow, the (total) number of bytes in those packets, the timestamp of the first and last packet, and protocol flag information such as whether any of those packets had the SYN flag set. NetFlow uses four rules to decide when to remove a flow record from router memory and report it to the collection station: 1) when TCP flags (FIN or RST) indicate flow termination, 2) 15 seconds (configurable "inactive timeout") after seeing the last packet with a matching flow ID, 3) 30 minutes (configurable "active timeout")' after the record was created to avoid staleness and 4) when the memory is full.

On every new packet, NetFlow looks up the corresponding entry (creating a new entry if necessary) and updates that entry's counters and timestamps. Since for high speed interfaces, the processor and the memory holding the flow records cannot keep up with the packet rate, Cisco introduced Sampled NetFlow [22] which updates the flow cache only for sampled packets. For a configurable value of a parameter $N$, a packet is sampled with one in $N$ probability.

One problem with NetFlow is that the memory required by the flow records and the bandwidth consumed to report them depends strongly on the traffic mix. In particular, large floods of small packets with randomly spoofed source addresses can increase memory and bandwidth requirements by orders of magnitude. Adaptive NetFlow [10] solves this problem by dynamically adapting the sampling rate. Adaptive NetFlow divides the operation of the flow measurement algorithm into equally spaced time bins. Within each bin, the algorithm starts by sampling aggressively (high sampling probability). If memory is consumed too quickly, it switches to less aggressive sampling. It then "renormalizes" existing entries so that they reflect the counts they would have had with the new sampling rate in effect from the beginning of the bin. At the end of the bin, all entries are reported.

Using fixed size bins in Adaptive NetFlow increases the memory utilization compared to Sampled NetFlow and causes bursts in reporting bandwidth. Memory utilization is higher because, to operate seamlessly between binboundaries, Adaptive NetFlow requires two sets of records (double-buffering), one for current bin and one for records in the previous bin while they are being transmitted. Without double-buffering, flow records that expire at the binboundary need to be transmitted immediately to create space for the next set of entries. Large flows spanning multiple bins are reported separately for every bin increasing the bandwidth usage. Table 1 gives a summary comparison of Sampled NetFlow, Adaptive NetFlow and Flow Slices.

The flow records are used to estimate the number of bytes or packets in various traffic aggregates of interest. This can give network operators information about dominant applications, the network usage of various clients, traffic matrices, and many other useful statistics [12, 19, 1, 14]. Smart Sampling [8] is a way of reducing the data used by such analyses without significantly affecting their results. Smart Sampling retains flow records with probability proportional to the size of their byte counter. The flow records can also be used to estimate the number of active flows which is important when looking for denial of service attacks, scans, and worms in the traffic mix. Unfortunately, 
if we use Sampled NetFlow it is impossible to recover the number of flows in the original traffic from the collected data [5] unless we use protocol information. By using the SYN flag information in flow records we can accurately estimate the number of TCP flows in the traffic mix [9].

\section{Description of flow slices}

The core flow slicing algorithm is based on the sample and hold algorithm [11]. After presenting the core algorithm, we discuss four extensions: adding packet sampling to scale to high speed links, using an inactivity timeout to reduce memory usage at a router, adding binned measurement to reduce binning error during analysis, and adding multi-factor smart sampling to control the volume of flow data reported. The version of Flow Slices described used for Table 1 has the first two extensions. We also discuss the configuration parameters of Flow Slices, and how they can be set adaptively based on the current traffic mix.

\subsection{Core algorithm}

The core flow slicing algorithm addresses the problem of reducing the memory usage of the flow measurement module. Sampled NetFlow and Adaptive NetFlow use random packet sampling: they only handle sampled packets. Just as sample and hold [11], flow slicing uses sampling only to control the creation of flow entries, once a sampled packet creates an entry for a flow, all its subsequent packets are counted (not just the sampled ones). This increases the accuracy of the estimates of packet counts, without changing the memory requirement. We use the "flow slicing probability" $p$ to control the creation of flow entries. We expire and report each entry exactly $t$ seconds after its creation, irrespective of the rate at which packets arrive for a particular flow. We call this core algorithm "flow slicing" because each entry tracks a "slice" of length $t$ from the flow. Just as in the case of NetFlow, the entry associated with a flow has a byte and packet counter updated at every packet, timestamps for the first and last packet, and it stores protocol information such as whether any of the packets counted against the entry had the SYN flag set. To ensure unbiasedness of estimators, on creation of an entry we do not initialize the byte counter to the number of bytes $b_{\text {first }}$ in the packet that caused the creation of the entry, but to $b_{\text {first }} / p$ (see Section 4.2 for more details).

The slice length $t$ is related to the "active timeout" of NetFlow which controls for how long an active entry is kept before expiring and being reported (default 30 minutes). Both of these parameters limit the staleness of the data (i.e. if we have a long-lived flow, we know that its traffic will be reported with at most this much delay).

By dynamically adapting the flow slicing probability, we can control the rate at which entries are created and freed, thus ensuring that the algorithm stays within its allocated memory budget $M$. By keeping the rate at which entries

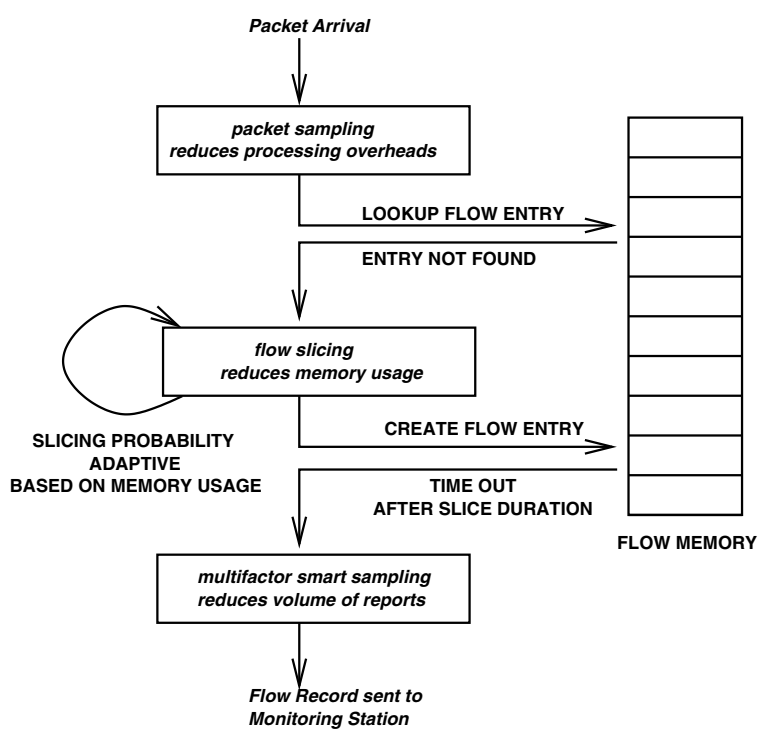

Figure 1: Architecture

are created, on average slightly below $M / t$, we can also keep the rate at which flows records are reported smooth. In contrast Adaptive NetFlow proposes expiring all active entries at the end of the measurement bin, so it either has a large peak in reports, or it requires buffers that increase the memory usage by almost a factor of two if the reporting of the records is smoothed out over the next measurement bin. We do not however, discuss dynamic adaptation in much detail in this paper, as adaptation techniques similar to that in [10] can be applied in this context using feedback from the current memory usage. Note however, that in our adaptation, we do not require the costly operation of renormalization that is required in Adaptive NetFlow. Next we discuss some of the tuning knobs we provide to control the three resource bottlenecks (CPU, Memory, Bandwidth).

\subsection{Scaling to high speeds}

The flow slicing probability $p$ controls the memory usage, but since we do a lookup in the flow memory for every packet, flow slicing does not control the processing load. In the presence of limited processing power, we add a random packet sampling stage in front of the flow slicing stage (see Figure 1). A simple solution is to set the packet sampling probability $q$ statically to a value that ensures that the processor performing the flow measurement can keep up even with worst case traffic mixes. Based on Cisco recommendations [17] for turning on NetFlow sampling for speeds higher than OC-3, we set $q$ to $1 / 4$ for OC-12 links, $1 / 16$ for OC-48, etc. With these packet sampling rates, and with worst case traffic consisting of the link entirely full with 40-byte packets, the flow measurement module has around $2 \mu s$ per packet and it has time to perform around 35 (wide) DRAM accesses on average. 


\subsection{Adding an inactivity timer}

Most flows in the Internet are short-lived. If our only mechanism for removing an entry is its expiration after the slice length $t$ and we use a large value for $t$, at any moment in time, most of the entries in the flow memory will belong to flows that are no longer active and just use up memory waiting to expire. On the other hand having a very short slice length can lead to an increase in reporting traffic and loss of accuracy. Adding an inactivity timeout parameter $t_{\text {inactive }}$ to flow slices reduces the memory spent on obsolete entries. Experimental results in Section 6.1 show that we can significantly reduce the memory requirement by using inactivity timers. An adaptive algorithm for setting the flow slicing rate can turn this reduction in memory usage into an increase in accuracy.

\subsection{Adding binned measurement}

With flow slices we have the same problem as with NetFlow if we want to perform traffic analysis using time bins: for flow slices that span time bins, we can only guess how many of the flow's packets were in each bin, and this introduces errors in the results. This problem is even more pronounced when analysis is required in very small time bins to capture more precise traffic dynamics. We can extend flow slices to support binned measurement of traffic by keeping multiple sets of byte and packet counters, one set for each bin the slice passes through. By keeping separate counters for each bin, the binning error is eliminated entirely, at the cost of increasing the size of the flow records. Note that the reporting bandwidth costs of this solution are significantly smaller than those of the solution used by Adaptive NetFlow where an entire record is reported for each bin. The byte and packet counters are 8 bytes whereas a complete record is 48 bytes.

The number of counters per record has to be one larger than the number of bins required to fit a slice because the flow slice can overlap only partially with the first and last bin. The choice of the size of the measurement bin supported is a compromise between resource consumption at the router and accuracy of results. Reasonable choices can range anywhere from the slice length $t$ to 20 times smaller. For brevity, we do not explore this further in the paper, but note that depending on the final goal, the flow slicing algorithm can be extended with additional resources to obtain the desired accuracy.

\subsection{Controlling the reporting bandwidth}

Smart sampling has been proposed as a way of reducing the number of flow records without causing much error. Smart sampling focuses on measuring the number of bytes in arbitrary aggregates of traffic and thus smart sampling favors flow records with large byte counters over those with small flow counters. Common packet sizes vary between 40 and 1500 , so while the packet counts are not proportional to the byte counts, they are closely correlated. Thus smart sampling will ensure that the errors introduced in packet counts are also small. The situation is different with flow arrival counts. These depend heavily on flow records with the SYN flag set, and most such records come from small flows which are discriminated against by smart sampling. Thus the errors introduced by smart sampling in the flow arrival counts are significant.

We propose a new variant of smart sampling, multifactor smart sampling which takes into consideration not just byte counts, but also packet counts and SYN flags. While multi-factor smart sampling still favors flow records with large byte and packet counts, it also favors records with the SYN flag, thus ensuring that the errors introduced into the flow arrival counts are not large either. Because the exact rule used to determine the multi-factor smart sampling probability $r$ depends on estimators of byte and packet counts, we postpone its discussion to Section 4.5.

\subsection{Setting the parameters of flow slicing}

Routers or other network devices performing flow measurement have three types of resources that can become bottlenecks: processing power, flow memory, and reporting bandwidth. Flow slices use three different "tuning knobs" to control these three resources: the packet sampling probability $q$ controls the processing load, the flow slicing probability $p$ controls the memory usage and the thresholds determining the smart sampling probability $r$ control the volume of data reported. This can result in more accurate traffic analysis results than using a single parameter, the packet sampling probability, to control all three resources, as Adaptive NetFlow does. This distinction would be irrelevant in practice if the only scarce resource would be the processing power at the router, so it is useful to perform a quick sanity check before proceeding any further: can an unfavorable traffic mix push the memory requirements or reporting bandwidth so high that they become a problem? First, let us assume a traffic mix consisting of back-to-back minimum sized packets, each belonging to a different flow (a massive flooding attack with randomly spoofed source addresses). With the packet sampling rates from Section 3.2, the traffic measurement module would receive a packet every $2 \mu \mathrm{s}$. Even with an aggressive inactivity timeout of $t_{\text {inactive }}=5$ seconds, we need a flow memory that can fit 2,500, 000 flow records, which at 64 bytes/record[17] requires 153 megabytes. When reported flow records take 48 bytes (ignoring overheads), so at 500,000 flow records/second, which requires 192 megabits/second. These numbers are orders of magnitude above what one can comfortably afford. The experiments from Section 6 use realistic traffic mixes to evaluate the benefits of Flow Slices as compared to Sampled NetFlow and Adaptive NetFlow.

For each of the parameters of Flow Slices listed in 


\begin{tabular}{|l|c|l|}
\hline Parameter & What it controls & How it is set \\
\hline \hline Flow slicing probability & Memory usage at router & Adaptively based on memory usage \\
\hline Flow slice length & Staleness of reported data & Statically based on user preferences \\
\hline Inactivity timeout & Reduces memory usage & Statically based on typical inter packet arrival time \\
\hline Packet sampling probability & Processing load at router & Statically based on worst case traffic \\
\hline Bin size (optional) & Binning error & Statically based on user preferences \\
\hline Smart sampling thresholds & Volume of flow data reported & Adaptively or statically based on target volume \\
\hline
\end{tabular}

Table 2: Configuration parameters for Flow Slices.

Table 2, we need to decide whether to set them statically as part of the router configuration, or dynamically adapt them to the current traffic mix. Of the three main tuning knobs, the flow slicing probability $p$ should definitely be set dynamically to allow the router to protect from memory overflow when faced with unfavorable traffic mixes. The thresholds controlling the smart sampling probability can also be set adaptively. In this paper, we consider that the packet sampling probability $q$ is static based on recommended values for different link capacities. Flow Slices would work just as well with a dynamic packet sampling probability that could go above the conservative static value, but since it is hard to guarantee the stability of such an approach without pushing the packet sampling rate adaptation logic into hardware (which raises deployment problems), we chose not to explore such a solution here.

The observant reader might have noticed that without the optional binned measurement feature Flow Slices resembles Sampled NetFlow. If the dynamic adaptation algorithms set the flow slicing probability $p$ and the smart sampling probability $r$ to 1 the two solutions perform exactly the same processing. We consider this to be an important feature. The difference between Sampled NetFlow and Flow Slices is in how they react to unfriendly traffic mixes and environments with strong constraints on resources. While both Adaptive NetFlow and Flow Slices provide robustness to unfavorable traffic mixes, Adaptive NetFlow forces the user to adopt the binned measurement model (which can increase memory usage and the volume of reports) even when the traffic mix is favorable.

\section{Estimators based on flow slices}

In this section, we discuss formulae for estimating traffic based on the flow records provided by Flow Slices. In practice, the user would be interested in the number of bytes, packets or flows in the entire traffic mix or a portion of it (e.g. the HTTP traffic, etc.). All our estimators focus on a single flow. To compute the total traffic, the user has to sum the contributions of all individual flow records. If the estimators for individual flows have the property of unbiasedness, the errors in the estimates for individual flows will not accumulate, but cancel out (to some extent).
For the purposes of our analysis, a bin is an arbitrary interval of time of interest to traffic analysis. To simplify analysis, we start by focusing on the simple case of a single bin, with slice length $t$ and inactivity timeout $t_{\text {inactive }}$ larger than the size of the bin and flow memory empty at the beginning of the bin. Next, we look at how the estimators generalize when we remove these constraints. Table 3 summarizes notation used throughout the paper.

\subsection{Estimating packet counts}

The packet counter $c_{s}$ in an entry is initialized to 1 when the first packet of the flow gets sampled, and it is incremented for all subsequent packets belonging to the flow. Let $s$ be the number of packets in the flow at the input of the flow slicing algorithm. Equation 1 gives the formula for our estimator $\widehat{s}$ for the number of packets in the flow.

$$
\widehat{s}=1 / p-1+c_{s}
$$

Lemma $1 \widehat{s}$ as defined in Equation 1 is an unbiased estimator of $s$.

Proof: By induction on the number of packets $s$.

Base case: If $s=1$, the only packet of the flow is sampled with probability $p$ and in that case it is counted as $1 / p-1+1=1 / p$ packets. With probability $1-p$ it is not sampled (and it counts as 0 ). Thus $E[\widehat{s}]=p \cdot 1 / p+0=$ $1=s$.

Inductive step: By induction hypothesis, we know that for a flow with $s^{\prime}=s-1, E\left[\hat{s}^{\prime}\right]=s^{\prime}=s-1$. Also since the flow slice length $t$ and the inactivity timeout $t_{\text {inactive }}$ are larger than the bin size, we know that once the flow gets an entry, all its packets within the bin will get counted by $c_{s}$. There are two possible cases: the first packet of the flow gets sampled, and we get $c_{s}=s$, or it doesn't and then the value of $c_{s}$ and $\widehat{s}$ will be the same as those for a flow with $s^{\prime}=s-1$ packets for which the sampling decisions are the same as for the rest of the packets of our flow.

$$
\begin{aligned}
E[\widehat{s}] & =p \cdot(1 / p-1+s)+(1-p) E\left[\widehat{s^{\prime}}\right] \\
& =1-p+p s+(1-p)(s-1)=s
\end{aligned}
$$




\begin{tabular}{|c|l|}
\hline Name & Meaning \\
\hline \hline$p$ & flow slicing probability \\
\hline$q$ & packet sampling probability \\
\hline$r$ & smart sampling probability \\
\hline$s$ & size of flow (in packets) before flow slicing \\
\hline$c_{s}$ & packet counter in flow record \\
\hline$\widehat{s}$ & estimate of the size of flow before flow slicing ( 0 if flow not sliced) \\
\hline$S$ & original size of flow (in packets) before packet sampling \\
\hline$\widehat{S}$ & estimate of the original size of flow (0 if flow not sampled or not sliced) \\
\hline$b$ & size of a flow in bytes before flow slicing \\
\hline$c_{b}$ & byte counter in flow record \\
\hline$\widehat{b}$ & estimate of the number of bytes in flow based on flow slices (0 if flow not sliced) \\
\hline$B$ & original size of flow in bytes before packet sampling \\
\hline$\widehat{B}$ & estimate of the original size of flow in bytes (0 if flow not sampled or not sliced) \\
\hline$\widehat{f}$ & contribution to the estimate of the number of active flows $(0$ if flow not sliced) \\
\hline$\widehat{a}$ & contribution to the estimate of the number of flow arrivals $(0$ if flow not sliced) \\
\hline$\widehat{A}^{(1)}$ & contribution to first estimator of number of flow arrivals $(0$ if flow not sampled or not sliced) \\
\hline$\widehat{A}{ }^{(2)}$ & contribution to second estimator of number of flow arrivals $(0$ if flow not sampled or not sliced) \\
\hline$z_{s}$ & smart sampling threshold controlling the influence of $\widehat{S}$ on $r$ \\
\hline$z_{b}$ & smart sampling threshold controlling the influence of $\widehat{B}$ on $r$ \\
\hline$z_{a}$ & smart sampling threshold controlling the influence of $\widehat{A}(1)$ on $r$ \\
\hline
\end{tabular}

Table 3: Notation used in this paper.

If we sample packets randomly with probability $q$ before applying the flow slicing algorithm, we will want to estimate the number of packets $S$ at the input of the packet sampling stage. Since $E[s]=q S$, it is easy to show that $\widehat{S}=1 / q \widehat{s}$ is an unbiased estimator for $S$.

\subsection{Estimating byte counts}

Before discussing how to estimate byte count estimates in flow slices, we show why a simpler solution does not work. We could have the byte counter $c_{b}$ in the flow entry just count the total number of bytes in the packets seen once the flow record is created. Just like with the packet counter, we need an additive correction to account for the packets missed before the creation of the entry. We can get an unbiased estimate for the number of packets missed, but not for their total size, because we do not know their sizes. We could assume that the packet sizes are uniform within the flow, but this would lead to systematic biases because they are not. As the proof of Lemma 2 shows, storing the size of the sampled packet that led to the creation of the entry would solve the problem because using it to estimate the total number of bytes in the packets not counted does lead to an unbiased estimator. But this would require another entry in the flow record. Instead, we store this information in the byte counter itself by initializing $c_{b}$ to $b_{\text {first }} / p$ when the entry is created $\left(b_{\text {first }}\right.$ is the size in bytes of the sampled packet). Let $b$ be the number of bytes of the flow at the input of the flow slicing algorithm.

$$
\widehat{b}=c_{b}
$$

Lemma $2 \widehat{b}$ as defined in Equation 2 is an unbiased estimator of $b$.

Proof: By induction on the number of packets in the flow $s$. Let $b_{i}$ for $i$ from 1 to $s$ be the sizes of the individual packets. By definition the number of bytes in the flow is $b=\sum_{i=1}^{s} b_{i}$. For convenience of notation, we index the packet sizes in reverse order, so $b_{1}$ will be the size of the last packet and $b_{s}$ the size of the first one.

Base case If $s=1$, the only packet is sampled with probability $p$ and in that case it is counted $c_{b}=b_{1} / p=b / p$ bytes. With probability $1-p$, it is not sampled (and it counts as 0 ). Thus $E\left[c_{b}\right]=p \cdot b / p+0=b$.

Inductive step By induction hypothesis, we know that if the first packet is not sampled we are left with the last $s^{\prime}=s-1$ packets and $E\left[c_{b}\right]=b^{\prime}=b-b_{s}$. If the first packet gets sampled, we count it as $b_{s} / p$ and we count the rest exactly because the flow slice length $t$ and the inactivity timeout $t_{\text {inactive }}$ are larger than the bin size.

$$
\begin{aligned}
E\left[c_{b}\right] & =p \cdot\left(b_{s} / p+b^{\prime}\right)+(1-p) b^{\prime} \\
& =b_{s}+p b^{\prime}+(1-p) b^{\prime}=b_{s}+b^{\prime}=b
\end{aligned}
$$

If we sample packets randomly with probability $q$ before applying the flow slicing algorithm, we will want to 
estimate the number of bytes $B$ at the input of the packet sampling stage. Since $E[b]=q B$, it is easy to show that $\widehat{B}=1 / q \widehat{b}$ is an unbiased estimator for $B$.

\subsection{Estimating the number of active flows}

We use two definitions for counting flows: active flows and flow arrivals. A flow is active during a time bin if it sends at least one packet during that time bin. Consecutive TCP connections between the same two computers that happen to share the same port numbers are considered a single flow and they will be reported in the same flow record under our current assumptions. Active flows with none of their packets sampled by the flow slicing process, will have no records; at least some of the flow records we get should be counted as more than one active flow, so that the total estimate will be unbiased. We count records with a packet counter $c_{s}$ of 1 as $1 / p$ flows and other records as 1 flow and this gives us unbiased estimates for the number of active flows.

$$
\widehat{f}= \begin{cases}1 / p & \text { if } c_{s}=1 \\ 1 & \text { if } c_{s}>1\end{cases}
$$

Lemma $3 \widehat{f}$ as defined in Equation 4 has expectation 1.

Proof: There are three possible cases: if a packet before the last gets sampled, $c_{s}>1$, if only the last packet gets sampled $c_{s}=1$, and if none of the packets gets sampled there will be no flow record, so the contribution of the flow to the estimate of the number of active flows will be $\widehat{f}=0$. The probability of the first case is $p_{s-1}=1-(1-p)^{s-1}$, the probability of the second is $p\left(1-p_{s-1}\right)$ and that of the third is $(1-p)\left(1-p_{s-1}\right)$.

$$
\begin{aligned}
E[\widehat{f}]= & p_{s-1} \cdot 1+p\left(1-p_{s-1}\right) \cdot 1 / p+ \\
& (1-p)\left(1-p_{s-1}\right) \cdot 0=1
\end{aligned}
$$

The estimators for the number of bytes and packets in a flow were trivial to generalize to the case where we apply random packet sampling before flow slicing because the expected number of packets and bytes after packet sampling was exactly $q$ times the number before. For the number of active flows there is no such simple relationship and actually it has been shown that it is impossible to estimate without significant bias the number of active flows once random sampling has been applied [5]. But by changing slightly the definition of flow counts we can take advantage of the SYN flags used by TCP flows.

\subsection{Estimating flow arrivals}

Flow arrivals are defined only for TCP flows which should start with one SYN packet. A flow is considered to have arrived in a bin if its SYN packet is in that time bin. Flows active during a certain bin, but with their SYN packet before the bin do not count as flow arrivals for that bin (but they count as active flows). If we look a the core flow slicing algorithm we can use the following estimator to compute the number of flow arrivals.

$$
\widehat{a}= \begin{cases}1 / p & \text { if SYN flag set } \\ 0 & \text { if SYN flag not set }\end{cases}
$$

Given that the SYN flag is set in the flow record if it was set in any of the packets counted against the record, it is trivial to prove that $\widehat{a}$ leads to unbiased estimates of the number of flow arrivals if we make an assumption.

Assumption 1 Only the first packet for the flow can have the SYN flag set.

The flow arrival information is preserved by random packet sampling. Duffield et al. propose two estimators of the number of flow arrivals that work based on flow records collected after random sampling of the traffic [9]. The formulas for the individual contributions of flow records to the total estimate of the number of flow arrivals are as follows.

$$
\begin{aligned}
& \widehat{M}^{(1)}= \begin{cases}1 / q & \text { if SYN flag set } \\
0 & \text { if SYN flag not set }\end{cases} \\
& \widehat{M}^{(2)}= \begin{cases}1 / q & \text { if SYN flag set and } s=1 \\
1 & \text { if SYN flag not set or } s>1\end{cases}
\end{aligned}
$$

Duffield et al. show [9] that both estimators are unbiased $E\left[\widehat{M}^{(1)}\right]=E\left[\widehat{M}^{(2)}\right]=1$ for flows that have exactly one SYN packet. Both estimators overestimate the number of flow arrivals if flows have more than 1 SYN packet. For flows without any SYN packets which according to our definition of flow arrivals (which differs slightly from that used in [9]) should not be counted, we have $E\left[\widehat{M}^{(1)}\right]=0$ and $E\left[\widehat{M}^{(2)}\right]>0$, so to make the second estimator unbiased we need another assumption.

Assumption 2 The first packet within the bin for every flow has the SYN flag set.

Flows retaining SYN packets after the random packet sampling stage will retain a single SYN packet, and $\widehat{M}^{(1)}$ estimates the number of flow arrivals based on the number of such flows. We can easily combine it with $\widehat{a}$ to get an estimator for the number of flow arrivals for the combined algorithm using random packet sampling and flow slicing.

$$
\widehat{A}^{(1)}= \begin{cases}1 /(p q) & \text { if SYN flag set } \\ 0 & \text { if SYN flag not set }\end{cases}
$$

$\widehat{M}^{(2)}$ treats separately flows that only have a SYN packet after packet sampling and the others that survive it. Fortunately we can differentiate between the two types of flows even after flow slicing is applied: if a flow with a single 
SYN packet is sampled by flow slicing its record will have $c_{s}=1$ and the SYN flag set; if any other flow is sampled by flow slicing and it has $c_{s}=1$ at the end of the bin it means that only its last packet was sampled thus it will not have the SYN flag set because that would put it into the category of flows with a single SYN packet surviving the packet sampling. Thus we can combine $\widehat{M}^{(2)}$ with $\widehat{a}$ to obtain another estimator.

$$
\widehat{A}^{(2)}= \begin{cases}1 /(p q) & \text { if SYN flag set and } c_{s}=1 \\ 1 / p & \text { if SYN flag not set and } c_{s}=1 \\ 1 & \text { if SYN flag not set and } c_{s}>1\end{cases}
$$

Note that if assumption 1 is violated and we have more than one SYN packet at the beginning of the flow, say due to SYN retransmissions, both estimators will be biased towards over-counting. But if repeated SYNs are a rare enough occurrence, the effect on a final estimate based on many flow records will be small.

\subsection{Multi-factor smart sampling}

To reduce the number of flow records, while maintaining accurate byte counts, smart sampling [8] proposes sampling the flow records with a size dependent probability $r=\min (1, b / z)$ where $z$ is a threshold parameter controlling the trade-off between the loss in accuracy and the reduction in the volume of reports. We can adapt smart sampling to flow slices using $r=\min (1, \widehat{B} / z)$ and we could still estimate byte, packet and flow arrival counts based on the smart sampled flow records using $\widehat{\mathcal{S}}=1 / r \widehat{S}$, $\widehat{\mathcal{B}}=1 / r \widehat{B}$, and $\widehat{\mathcal{A}}=1 / r \widehat{A}$. But using this formula for $r$ results in a variance for $\widehat{\mathcal{A}}$ much larger than that of $\widehat{A}$ because it discriminates against flows with few bytes, and since most flows have few bytes, they will also produce most flow records with the SYN flag set - and these are exactly the records $\widehat{A}^{(1)}$ and $\widehat{A}^{(2)}$ rely on.

We propose a new variant of smart sampling, multifactor smart sampling, which takes into consideration not just byte counts, but also packet counts and SYN flags. By picking a smart sampling probability of $r=\min \left(1, \widehat{s} / z_{s}+\right.$ $\left.\widehat{B} / z_{b}+\widehat{A} / z_{a}\right)$ we can balance the requirements of the three estimators. The three individual thresholds control the trade-off between accuracy and reduction in report volume separately for the three estimators of bytes, packets and flow arrivals. Note that multi-factor smart sampling is a generalization of smart sampling: if we set $z_{b}=z$, $z_{s}=\infty$, and $z_{a}=\infty$, it will assign the exact same sampling probabilities to records as smart sampling.

\subsection{Dynamically adjusting the flow slicing probability}

Flow Slices dynamically adjusts the flow slicing probability $p$ to the current traffic. This adjustment can happen in the middle of a time bin. Which one of the many values of $p$ should we use in our estimators? Are the estimators still unbiased? Actually none of the proofs depends on having a single value for $p$, and they would all work if we replaced it with a separate $p_{i}$ for every packet. All the estimators would need to use the value of the packet slicing probability in effect at the time the sampling of a packet caused the creation of the entry. This doesn't necessarily mean that one needs to extend the flow entry with one more field, because it already holds the timestamp of the first packet and that can be used to determine the flow slicing rate if the router keeps a small log of recent adjustments to it.

When the flow record expires and it is reported, the report should include the value of the flow slicing probability $p$ in effect at the time the entry was created. Similarly if the smart sampling thresholds $z_{s}, z_{b}$, and $z_{a}$ are adjusted dynamically, the report should include their current value so that one can compute $r$ during analysis. If one uses just a few possible values for these parameters (e.g. only powers of two), each of these sampling rates can be encoded in less than one byte, so the reporting overhead they impose is limited (a flow record has 48 bytes).

\subsection{Bins, timeouts, and flow reconstruction}

To simplify our discussion of the estimators we started with some strong assumptions: all records last longer than the bin length, counters count only packets within the bin of interest, and the flow memory is empty at the beginning of the bin. In this section we relax these assumptions and discuss the effects of these relaxations on the estimators.

\subsubsection{Continuous operation}

The most elementary relaxation of the assumption is to consider continuous operation of the algorithm: records still last longer than the bin length, and we still have separate counters for each bin, but there can be active records at the start of our bin, records created earlier.

The simplest case is that of records spanning the entire bin. The byte and packet counters will reflect the actual traffic, so we use $\widehat{S}=1 / q c_{s}$ and $\widehat{B}=1 / q c_{b}$. If we do not have a packet sampling stage we can also compute $\widehat{f}=1$ if $c_{s}>0$ and $\widehat{f}=0$ otherwise. $\widehat{A}=0$ because the flow started in an earlier bin.

If a flow record expires within the bin we run the analysis on, it can be the only record for the flow, but it is also possible that another record for the same flow would get created after the first record's expiration. For byte and packet counts which are additive we can just add the counters from the first record to the estimates from the second $\widehat{s}=\widehat{s}_{1}+\widehat{s}_{2}$ and $\widehat{b}=\widehat{b}_{1}+\widehat{b}_{2}$. The analysis of unbiasedness carries through because we can consider that the bin is actually two sub-bins, one ending when the first record ends and the other starting at the same time. Since we have unbiased byte and packet estimates for both sub-bins, our estimates for the sum of the bins will still be unbiased. 
If $c_{s 1}>0$, we know that the flow sent packets during the bin, so we set $\widehat{f}$ to 1 , otherwise we use Equation 3 with $c_{s 2}$ since an unbiased estimator for whether the flow was active in the second sub-bin will tell use whether it was active overall. This approach preserves overall unbiasedness, but it makes analysis more complicated because the two flow records representing the flow cannot be processed independently anymore: the contribution of the second record to the flow count of the bin depends on whether there was a first record with the same flow identifier. When the router reports the records, they might not be near each other, so the analysis has to do "flow reconstruction": keep a hash table with flow identifiers and find flow records with the same flow identifier covering parts of the same bin. The consequence of not doing flow reconstruction is running the risk of double counting such flows with more than one record (which might be acceptable in many settings).

By our definition of flow arrivals from Section 4.4, as long as assumption 1 holds, if a flow has a record that starts before the start of the bin, we should use $\widehat{A}=0$, irrespective of whether we have a second flow record (possibly with a SYN flag) or not. If we have a second flow record with the SYN flag set we can clearly say that assumption 1 does not hold, but without flow reconstruction we might count it separately against the flow arrival count. In many settings this type of over-counting is not a serious concern. $\widehat{A}^{(2)}$ should not be used because assumption 2 does not hold.

\subsubsection{Slices shorter than bins}

When the inactivity timeout $t_{\text {inactive }}$ is short or when the analysis is over long time bins (say hours), flow slices can be shorter than the bin size. It can happen that we have more than two records for the same flow within the same bin. For byte and packet counts we can just add the individual estimates for the different records and we get an unbiased estimator for the entire bin. For active flows we cannot get an unbiased estimate, not even with flow reconstruction. For flow arrivals, by using $\widehat{A}^{(1)}$ for the individual records and summing the contributions without any flow reconstruction gives unbiased estimates as long as assumption 1 is not violated. For a record started before the beginning of the bin, even if it has the SYN flag set in violation of assumption 1 we do not count it as flow arrival and thus have $\widehat{A}^{(1)}=0$.

\subsubsection{Binning errors}

So far we assumed that Flow Slices uses binned measurement. This guarantees that as long as the analysis is on time intervals that are exact multiples of the measurement bins used, it will be easy to determine exactly how many of the packets and the bytes counted by the record were within the bin. But by default Flow Slices doesn't use bins, and for records that span bin boundaries, the user will have to guess how the packets and bytes were actually divided between the bins. We can prove that our reconstruction of how the traffic divides between the bins is unbiased only if we make an assumption about the spacing of the packets.

Assumption 3 For every flow at the input of the flow slicing algorithm, the time between the arrivals of all pairs of its consecutive packets is the same.

We use the following algorithm for distributing the packets of reported by a flow record that spans bins between the bins covered by the record. We consider $c_{s}$ packet arrival events, the first one is the timestamp of the first packet counted by the entry, the last one the timestamp of the last packet counted by the entry and the remaining $c_{s}-2$ evenly spaced between them. We consider that 1 packet arrived at every packet arrival event, except for the first event which has $1 / p$ packets, and distribute the packets between bins accordingly. This can be shown to be an unbiased way of distributing packets between bins under assumption 3 . We recommend distributing the $c_{b}$ bytes of the flow between bins proportionally with the number of packets counted against each bin. Assumption 3 is not enough to prove this distribution of bytes between the bins to be unbiased, we would need an additional assumption about uniformity of packet sizes. For flow arrivals, we do not have a binning problem because we assume that the first packet counted by the flow record is the one with the SYN, so we count the flow arrival against the bin the first packet is in.

We cannot achieve provably unbiased binning for bytes and packets under realistic assumptions about inter packet arrival times and packet size distributions within flows. We turn to measurements instead to see how much the binning error is on typical traffic. We recommend using such experimental results to decide whether increasing the size of the flow record by adding multiple counters to do binned measurement is worth it.

\section{Variances of estimators}

The estimators discussed in the previous section were all defined on an individual flow and to compute a measure (say the number of packets) for a larger aggregate, the analyst would sum the values of the estimators for the flow records matching the aggregate. The sampling decisions for different flows are fortunately independent and thus the variance of the estimates for aggregates are the sum of the respective variances for the estimators for individual flows. In this section we focus on studying the variances of the various estimators for individual flows. We also show that the variances of the estimators based on the core flow slicing algorithm are lower than those of estimators based on random sampling used by Adaptive NetFlow to control memory usage. As in Section 4, we start with a simplified setting of a single bin in isolation and then proceed to more realistic settings. The proofs for the variance results from this section can be found in technical report[15]. 


\subsection{Packet count variance}

For the core flow slicing algorithm we can compute the variance of the packet count estimator.

$$
V A R[\widehat{s}]=1 / p(1 / p-1)\left(1-(1-p)^{s}\right)
$$

Note how this variance is strictly lower than the variance of results based on random packet sampling $(1 / p-1) s$ except for the case of $s=1$ when the two variances are equal. The higher $s$, the larger the difference between the variance of results based on flow slicing when compared with packet sampling. Since using the same sampling probability will give the same memory usage for flow slicing and ordinary sampling, this comparison of variances shows us that flow slicing is a superior solution. The advantage is most apparent when estimating the traffic of aggregates with much traffic coming from large flows.

The same conclusion holds if we compare the combination of packet sampling and flow slicing used by Flow Slices to the pure packet sampling used by Adaptive NetFlow and Sampled NetFlow. Here the fair comparison is with Sampled NetFlow using a packet sampling probability of $p q$. We can conceptually divide this into a first stage of packet sampling that samples packets with probability $q$ and a second one that samples them with probability $p$. The first stage has identical statistical properties for the two solutions, thus the difference in the accuracy is given by the second stage, but comparing the second stages reduces to comparing flow slicing and packet sampling using the same probability $p$.

\subsection{Byte count variance}

We can also compute the variance of the estimates for the number of bytes (we number the packet sizes $b_{i}$ in reverse order with $b_{1}$ being the size of the last packet and $b_{s}$ that of the first one).

$$
V A R[\widehat{b}]=1 / p \sum_{i=1}^{s}(1-p)^{i-s+1} b_{i}^{2}
$$

Note how this variance is strictly lower than the variance of results based on random packet sampling $(1 / p-$ 1) $\sum_{i=1}^{s} b_{i}^{2}$ (except for the case of a single packet flow). This shows that for byte counts too, flow slices are a better solution than ordinary sampling.

\subsection{Flow count variance}

We can also compute the variance of the estimates for the number of active flows. We cannot compare against packet sampling because there are no unbiased estimates for the number of active flows based on packet sampled data.

$$
V A R[\widehat{f}]=(1-p)^{s-1}(1 / p-1)
$$

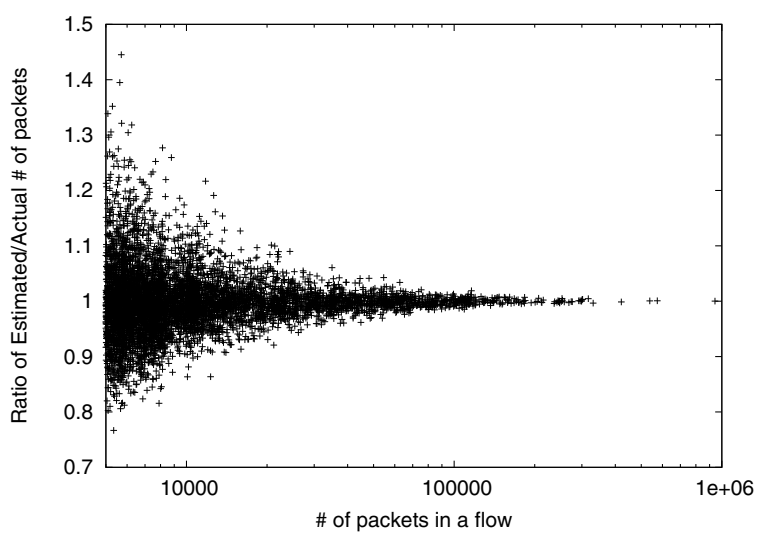

Figure 2: Scatter plot depicting the accuracy of packet count estimates based on flow slices.

\subsection{Continuous operation}

If we consider continuous operation for the algorithm, we can have at the beginning of the bin a record for our flow. If the slice spans the entire bin, it counts everything exactly and thus the variance of all estimator is 0 . If the slice ends in the current bin, we can divide the flow into two parts: one covered by this older record and the rest. For the first part we have 0 variance for the byte and packet counts and for the second part we can apply formulas 7 and 8 , but instead of $s$ being the number of packets of the flow in the bin, it should be only the number of packets in this second part and the $b_{i}$ be the sizes of those packets. For the flow count estimate, if the number of packets in the first record is 0 , the variance of the estimate is 0 , otherwise formula 9 applies. Thus having flow records active at the beginning of the bin does not increase the variance of the packet, byte and flow count estimates, on the contrary, it can reduce them significantly.

\section{Experimental evaluation}

We divide the experimental evaluation into two parts. The first group of experiments evaluates the efficacy of the core flow slicing algorithm and the multi-factor smart sampling algorithm. The second group compares the Flow Slices solution with Adaptive NetFlow (ANF) to show the efficacy of Flow Slices both in terms of memory usage and accuracy of estimates. For our evaluations, we obtained traces on an OC-48 link from CAIDA [4].

\subsection{Accuracy of core flow slicing algorithm}

In this section, we evaluate the core flow slicing algorithm against the "full-state" approach. These experiments provide more insight into the efficacy of the flow slicing algorithm and the effect of changing various variables such as flow slicing probability and slice length on the memory usage and the mean relative error of results. 


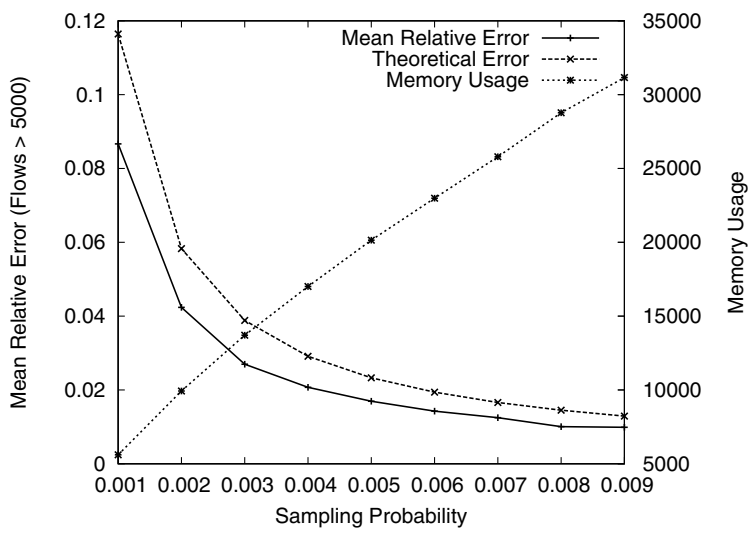

Figure 3: Trade-off between the mean relative error and memory usage as we increase flow slicing probability.

Are estimates unbiased? For this experiment, we fix the flow slicing probability $p$ to $0.8 \%$ (1 in 125$)$ and the slice duration to 60 seconds. Figure 2 shows the scatter plot of ratio of the estimated and true flow sizes (in number of packets) on the y-axis with increasing true flow size on the $\mathrm{x}$-axis. Note that the plot only shows flows that have more than 5,000 packets throughout the duration of the trace (1 hour). From this scatter plot, we can see that most of the flows have been accurately estimated (within 10\%). The estimates converge to the true values as the flow size increases. The presence of two-sided errors empirically confirms the unbiasedness of estimates based on flow slicing.

What is the effect of flow slicing probability on the accuracy of these estimates? According to Equation 7, increasing flow slicing probability increases the accuracy of estimated flow sizes. Besides, the memory usage should increase as the slicing probability increases. In Figure 3, the mean relative error for flows larger than 5,000 and the corresponding memory usage have been plotted with varying slicing probability on the $\mathrm{x}$-axis. Apart from the empirical value of the mean relative error, we also plot the theoretical value based on Equation 7. Figure 3 confirms that increasing slicing probability decreases the mean relative error while increasing the memory usage. It can also be observed from the figure that the theoretical and empirical values of mean relative errors are in close agreement thus validating the analysis in Section 5.1.

What kinds of errors do we introduce by interpolating the number of packets in time bins? The goal of this experiment is to study the errors introduced when interpolating the number of packets in various time bins from flow slices that do not use bins (they only store the timestamps of the first and last packet). In Figure 4, the y-axis has the ratio of estimated to actual size of the flow in a given bin and the $\mathrm{X}$-axis has the actual flow size (in packets). For this experiment, we used a slice length of 90 seconds and divided it up equally into 10 bins of size 9 seconds each. Two conclu-

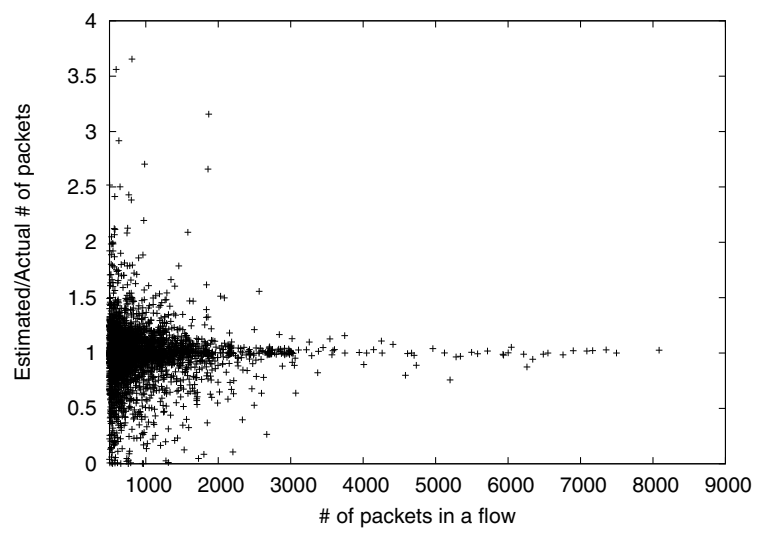

Figure 4: Scatter plot depicting the errors introduced in interpolating bin measures from slices.

sions can be drawn from the results in Figure 4. First, for large flows, the error in the estimates obtained by interpolating bins from slices is insignificant. On the other hand, for relatively small flows, interpolating from flow slices results in much higher error. This is because we divide the entire volume of traffic for a particular flow among the bins it covers (see Section 4.7.3 for more details); the error depends on the timing of bursts of traffic. Of course, to capture the fine grained traffic information, the extension proposed in Section 3.4 could be used, but it would result in higher memory requirements. Second, we can observe the presence two-sided errors indicates lack of bias.

What is the effect of multi-factor smart sampling on the accuracy of estimates? In Section 4.5, we proposed a modification to smart sampling to improve the accuracy of the estimates for the number of flow arrivals. Table 4 summarizes the results of our experiment comparing multi-factor smart sampling with smart sampling. Before we discuss the details of this experiment, we want to note that we found that Assumption 1, that only the first packet of a flow can have the SYN flag set, is often violated in our trace. For some applications, the average number of packets with the SYN flag set per flow is almost 2 (due to SYN retransmissions). This affects all estimators of flow arrivals based on SYN counts. In this experiment, we do not aim to evaluate the accuracy of estimators based on SYN counts, but the effect of smart sampling on their estimates. Therefore, we do not measure the error relative to the actual number of flow arrivals, but to the estimate of flow arrivals based on the input to the two smart sampling algorithms. The input we used is the result of Flow Slices with a packet sampling probability of $q=1 / 4$ and flow slicing probability of $p=1 / 4$, using a slice length of 60 seconds and an inactivity timeout of 15 seconds. The threshold used for smart sampling is $z=50,000$ bytes. The thresholds used for multi-factor smart sampling, $z_{s}=1,000$ packets, $z_{b}=500,000$ bytes and $z_{a}=50$ flows, have been selected so that it produces 


\begin{tabular}{|c|c|c|c|c|c|c|c|c|c|}
\hline Port number used as & \multicolumn{3}{|c|}{ Multifactor s. s. error } & \multicolumn{2}{|c|}{ Smart sampling error } & \multicolumn{3}{|c|}{ Actual traffic } \\
\cline { 2 - 10 } aggregation key & Pkts & Bytes & SYNs & Pkts & Bytes & SYNs & Pkts & Bytes & SYNs \\
\hline \hline Web (80) & $0.4 \%$ & $0.7 \%$ & $0.8 \%$ & $0.3 \%$ & $0.1 \%$ & $1.6 \%$ & $17.5 \mathrm{M}$ & $1582 \mathrm{M}$ & $1852 \mathrm{~K}$ \\
\hline Kazaa (1214) & $0.4 \%$ & $0.2 \%$ & $2.4 \%$ & $0.6 \%$ & $0.1 \%$ & $12.4 \%$ & $2.67 \mathrm{M}$ & $1527 \mathrm{M}$ & $44.9 \mathrm{~K}$ \\
\hline eDonkey (4662) & $0.5 \%$ & $0.7 \%$ & $1.5 \%$ & $1.0 \%$ & $0.2 \%$ & $4.5 \%$ & $2.96 \mathrm{M}$ & $1075 \mathrm{M}$ & $344 \mathrm{~K}$ \\
\hline telnet (23) & $0.6 \%$ & $0.8 \%$ & $4.9 \%$ & $0.9 \%$ & $1.0 \%$ & $39.2 \%$ & $1.84 \mathrm{M}$ & $79.1 \mathrm{M}$ & $12.0 \mathrm{~K}$ \\
\hline SMB (445) & $1.3 \%$ & $1.6 \%$ & $1.1 \%$ & $2.5 \%$ & $1.8 \%$ & $3.1 \%$ & $1.50 \mathrm{M}$ & $93.3 \mathrm{M}$ & $1380 \mathrm{~K}$ \\
\hline SMTP (25) & $1.9 \%$ & $1.0 \%$ & $1.4 \%$ & $2.7 \%$ & $0.9 \%$ & $6.4 \%$ & $0.43 \mathrm{M}$ & $130 \mathrm{M}$ & $86.9 \mathrm{~K}$ \\
\hline DNS (53) & $1.8 \%$ & $2.4 \%$ & $3.6 \%$ & $2.7 \%$ & $1.7 \%$ & $16.8 \%$ & $0.45 \mathrm{M}$ & $34.8 \mathrm{M}$ & $6.02 \mathrm{~K}$ \\
\hline
\end{tabular}

Table 4: Comparison of the error introduced by multifactor smart sampling and smart sampling into estimates of traffic of various applications (average of 10 runs with different seeds). Both algorithms were configured to reduce the number of flow records from 1,700,000 to around 190,000.

\begin{tabular}{|c|c|c|c|c|c|c|c|c|}
\hline \multirow{2}{*}{$\begin{array}{c}\text { Port number/ } \\
\text { Range }\end{array}$} & \multicolumn{2}{|c|}{ Adaptive NetFlow } & \multicolumn{2}{|c|}{ Flow Slices(60s) } & \multicolumn{2}{c|}{ Flow Slices(180s) } & \multicolumn{2}{c|}{ Flow Slices(300s) } \\
\cline { 2 - 9 } & Packets & Bytes & Packets & Bytes & Packets & Bytes & Packets & Bytes \\
\hline \hline Web (80) & $0.5 \%$ & $1.4 \%$ & $0.4 \%$ & $2.0 \%$ & $0.5 \%$ & $1.5 \%$ & $0.3 \%$ & $0.9 \%$ \\
\hline Kazaa (1214) & $1.2 \%$ & $2.6 \%$ & $1.0 \%$ & $2.4 \%$ & $0.8 \%$ & $1.0 \%$ & $1.0 \%$ & $1.4 \%$ \\
\hline eDonkey (4662) & $1.4 \%$ & $3.3 \%$ & $1.7 \%$ & $2.1 \%$ & $1.1 \%$ & $1.9 \%$ & $1.0 \%$ & $1.9 \%$ \\
\hline telnet (23) & $1.3 \%$ & $1.5 \%$ & $2.2 \%$ & $2.2 \%$ & $2.1 \%$ & $1.8 \%$ & $2.3 \%$ & $2.5 \%$ \\
\hline SMB (445) & $2.6 \%$ & $3.3 \%$ & $2.5 \%$ & $5.0 \%$ & $2.2 \%$ & $2.5 \%$ & $1.7 \%$ & $4.1 \%$ \\
\hline SMTP (25) & $1.9 \%$ & $8.7 \%$ & $2.3 \%$ & $7.7 \%$ & $3.9 \%$ & $6.5 \%$ & $3.8 \%$ & $6.8 \%$ \\
\hline DNS (53) & $2.7 \%$ & $4.0 \%$ & $3.6 \%$ & $2.6 \%$ & $2.8 \%$ & $3.6 \%$ & $4.4 \%$ & $4.9 \%$ \\
\hline$>50,000$ & $9.7 \%$ & $10.1 \%$ & $5.4 \%$ & $5.6 \%$ & $3.7 \%$ & $3.9 \%$ & $3.1 \%$ & $3.3 \%$ \\
\hline $10,000-50,000$ & $20.5 \%$ & $21.9 \%$ & $15.3 \%$ & $16.7 \%$ & $12.2 \%$ & $13.5 \%$ & $10.8 \%$ & $11.9 \%$ \\
\hline $5,000-10,000$ & $30.6 \%$ & $35.8 \%$ & $26 \%$ & $29.4 \%$ & $22.2 \%$ & $24.9 \%$ & $19.9 \%$ & $22.5 \%$ \\
\hline
\end{tabular}

Table 5: Comparison of the accuracy of estimates based on Adaptive Netflow and Flow Slices with different slice lengths.

approximately same number of records as smart sampling (from 1,700,000 down to roughly 190,000). Table 4 shows the error introduced by the two variants of smart sampling into estimates of the traffic of various applications identified by destination port numbers. While smart sampling introduces very large errors in the flow arrival estimates, multi-factor smart sampling ensures that the errors are comparable to packet and byte count estimates. For example, smart sampling incurs an error of $39.2 \%$ for telnet because it's small flows (approximately 6,600 bytes per flow on average compared to 34,000 for Kazaa) are discriminated against by smart sampling. Multi-factor smart sampling, on the other hand, achieves more accurate flow arrival counts by biasing its sampling towards records with non-zero flow arrivals. This typically results in only a slight reduction in the accuracy of packet and byte count estimates.

\subsection{Comparison with Adaptive NetFlow}

In this section, we compare Flow Slices with Adaptive NetFlow [10], a previously proposed solution based on packet sampling. For the purposes of evaluation, we fix the packet sampling probability to 1 in 1024 for ANF. To be fair in our comparisons with Flow Slices, we split the 1/1024 proba- bility into two parts consisting of packet sampling (1/16 for our OC-48 trace) and flow slicing probability (1/64). We compare average error in the estimates for both individual flows (categorized by ranges) as well as aggregates based on destination port number. typically, are Table 5 shows that ANF and Flow Slices have similar errors when estimating the traffic of various applications (aggregated by port). However, Flow Slices performs better than ANF (by about $10 \%$ ) in the average error for individual flows. Varying the slice length from 60 to 300 seconds for Flow Slices did not affect the accuracy of the results significantly, although bigger slice lengths seem to perform a little better than with smaller slice lengths.

How does Flow Slices compare with ANF in resource consumption? Table 6 summarizes the memory usage at the router and the volume of traffic reports for Flow Slices and ANF. Without an inactivity timeout, the resource requirements of the two solutions are similar. As we move to longer bins/slices there is a slight decrease in report volumes and a significant increase in memory requirements. Adding an inactivity timeout of 15 seconds to Flow Slices has a dramatic effect. The memory requirements are reduced significantly (about $80 \%$ ) at the cost of only a slight increase in the volume of the reports (about 5\%). With the 


\begin{tabular}{|c|c|c|c|c|c|c|c|c|}
\hline Trace & Packets & Slice length / & \multicolumn{3}{|c|}{ Memory (entries) } & \multicolumn{3}{|c|}{ Report volume (records) } \\
\cline { 4 - 9 } & per second & Bin size (in secs) & Slices & $t_{\text {inactive }}$ & ANF & Slices & $t_{\text {inactive }}$ & ANF \\
\hline \hline 1 (1 hour) & 23,733 & 60 & 1,148 & 597 & 1,195 & 68,537 & 63,658 & 64,764 \\
\hline $1(1$ hour) & 23,733 & 180 & 3,021 & 741 & 3,141 & 61,316 & 57,028 & 60,229 \\
\hline $1(1$ hour) & 23,733 & 300 & 4,691 & 793 & 4,158 & 57,635 & 53,953 & 58,730 \\
\hline \hline $2(10$ mins) & 124,988 & 60 & 5,378 & 3,065 & 5,641 & 25,896 & 26,362 & 27,509 \\
\hline $2(10$ mins $)$ & 124,988 & 180 & 14,046 & 3,944 & 14,049 & 22,896 & 23,800 & 23,994 \\
\hline $2(10$ mins $)$ & 124,988 & 300 & 21,667 & 4,218 & 21,716 & 21,667 & 22,841 & 21,716 \\
\hline
\end{tabular}

Table 6: Comparison of the amount of memory used and the volume of traffic reports generated by Flow Slices and ANF for different slice lengths (in Flow Slices) and bin sizes (in ANF). The $t_{\text {inactive }}$ columns show the memory savings Flow Slices achieve by using an inactivity timeout of 15 seconds.
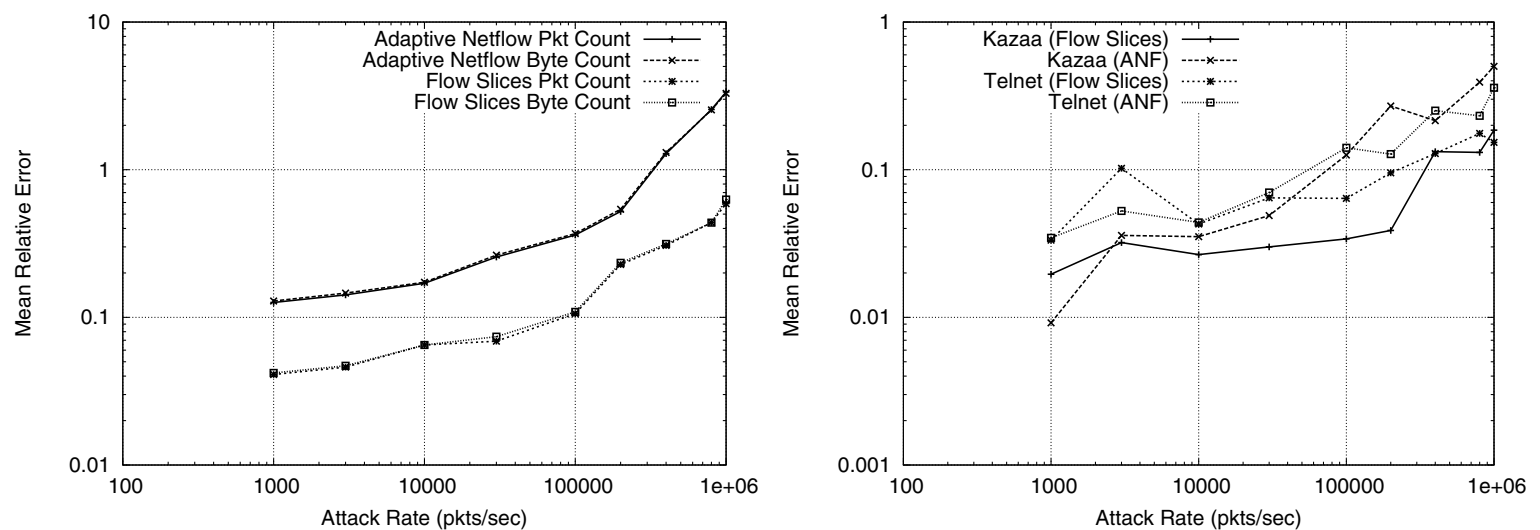

Figure 5: Accuracy of ANF and Flow Slices the rate of attack traffic increases. The left plot compares the average relative error in estimating the size of flows with more than 5,000 packets. The right plot compares the average relative error in estimating the size of two traffic aggregates - telnet and Kazaa. All results are averages over 10 runs with different seeds.

inactivity timeout, the memory usage of Flow Slices is less sensitive to the slice length. The lower memory usage of Flow Slices compared to ANF has important consequences when the sampling rates are adapted dynamically. Given the same memory constraints, the sampling rate adaptation algorithm can converge to more aggressive sampling rates for Flow Slices which results in more accurate estimates.

What is the effect of Denial-of-Service attacks? Figure 5 compares the estimates obtained by ANF and Flow Slices in the presence of a DoS attack. We varied the attack rate from 1000 packets-per-second (pps) to 1.6 million pps; each attack packet represents a different flow as source addresses are spoofed at random. We configured ANF and Flow Slices to operate within a memory budget of 8,000 flow records (not including the buffering needed by ANF to transmit the records at the end of the measurement bin). ANF converged to smaller sampling probabilities as attack traffic gained intensity; the sampling probability varied from $0.155 \%$ at 1,000 pps to $0.0026 \%$ at 1.6 million pps. Similarly, for Flow Slices, while the random packet sampling probability remained constant at $q=1 / 16$ (to simulate real hardware constraints), the combined sam- pling probability $p \cdot q$ varied from $0.781 \%$ to $0.0156 \%$. Flow Slices could afford more aggressive sampling mainly due to the use of an inactivity timeout of 15 seconds (the slice length for Flow Slices and bin size for ANF were $60 \mathrm{sec}-$ onds). On the left, we plot the attack rate on the x-axis and the mean relative error (both for packet and byte counts) of flows with more than 5,000 packets on the y-axis, both in log-scale. For comparable memory usage, in the presence of DoS attacks, Flow Slices produces traffic estimates an order of magnitude better than those of ANF. On the right, we plot the average relative error in estimating traffic that belongs to two different applications - telnet and Kazaa, using the two flow measurement solutions. While the accuracy of both the estimates reduces as the attack rate increases, Flow Slices provides better accuracy than ANF.

While these results do not prove that for all traffic mixes, Flow Slices perform better than other solutions, these results do show the efficacy of the Flow Slices on realistic traffic mixes. When we apply inactivity timeouts to the Flow Slices, it results in much better re-use of memory at the cost of a small loss in accuracy and a little increase in the total volume of flow records reported. 


\section{Conclusions and future work}

Processing, memory, and bandwidth constraints make it impossible for high speed routers to provide full flow measurements, thus forcing us to consider some type of data reduction. Different flow measurement solutions perform this data reduction differently, and one can compare them by comparing their resource consumption and the amount of error the data reduction causes in various analyses one wants to perform on the flow data. Flow Slices offers a unique mix of qualities among flow measurement solutions: dynamic adaptation of sampling parameters to keep resource usage within limits, separate parameters for controlling the three potential resource bottlenecks, efficient use of available resources, and algorithmic solutions for minimizing the errors introduced by the data reduction. These qualities are possible due to novel algorithms such as the core flow slicing algorithm and multi-factor smart sampling and various new estimators. Our experiments also confirm that compared to the currently used Sampled NetFlow and Adaptive NetFlow, Flow Slices constitutes a better flow measurement solution.

But the fact that Flow Slices supports well the traffic analyses discussed in this paper, does not mean there is no room for improvement. There are many useful analyses of unsampled flow data that we haven't considered. For instance, correlation across flows has been used in $[19,13]$ to classify different flows (such as control and data connections for the same FTP session) into one application. Additional metrics such as flow duration and the variability of packet inter-arrival times have been used to divide flows into different application categories [21]. Such analyses require additional information to be preserved by sampling techniques in order for them to be effective. It is an important challenge to adapt data reduction techniques such as sampling to enable such sophisticated traffic analyses.

\section{Acknowledgments}

We wish to thank Nick Duffield for discussions that contributed to the idea of the $\widehat{f}$ estimator and Ken Keys for helping us with the code of Adaptive NetFlow. We also wish to thank the anonymous reviewers whose comments helped greatly improve this paper. This work was made possible through NSF grant ANI0074004.

\section{References}

[1] Ipmon - packet trace analysis. http://ipmon.sprintlabs.com/ packstat/ packetoverview.php.

[2] N. Brownlee, C. Mills, and G. Ruth. Traffic flow measurement: Architecture. RFC 2722, Oct. 1999.

[3] N. Brownlee and D. Plonka. IP flow information export (ipfix). IETF working group.

[4] Cooperative association for internet data analysis. http://www.caida.org/.
[5] S. Chaudhuri, R. Motwani, and V. Narasayya. Random sampling for histogram construction: How much is enough? In SIGMOD, 1998.

[6] C. Cranor, T. Johnson, O. Spatschek, and V. Shkapenyuk. Gigascope: A stream database for network applications. In SIGMOD, June 2003.

[7] The DAG project. http://dag.cs.waikato.ac.nz/.

[8] N. Duffield, C. Lund, and M. Thorup. Charging from sampled network usage. In IMW, Nov. 2001.

[9] N. Duffield, C. Lund, and M. Thorup. Properties and prediction of flow statistics from sampled packet streams. In $I M W$, Nov. 2002.

[10] C. Estan, K. Keys, D. Moore, and G. Varghese. Building a better netflow. In Proceedings of the ACM SIGCOMM, Aug. 2004.

[11] C. Estan and G. Varghese. New directions in traffic measurement and accounting: Focusing on the elephants, ignoring the mice. In ACM Trans. Comput. Syst., Aug. 2003.

[12] A. Feldmann, A. Greenberg, C. Lund, N. Reingold, J. Rexford, and F. True. Deriving traffic demands for operational ip networks: Methodology and experience. In SIGCOMM, Aug. 2000.

[13] T. Karagiannis, A. Broido, M. Faloutsos, and K. claffy. Transport layer identification of $\mathrm{p} 2 \mathrm{p}$ traffic. In $I M C$, Oct. 2004.

[14] K. Keys, D. Moore, R. Koga, E. Lagache, M. Tesch, and $\mathrm{k}$ claffy. The architecture of CoralReef: an Internet traffic monitoring software suite. In PAM, Apr. 2001.

[15] R. R. Kompella and C. Estan. The power of slicing in internet flow measurement. Technical report, UCSD, May 2005.

[16] K. McCloghrie and M. T. Rose. Rfc 1213, Mar. 1991.

[17] Cisco NetFlow. http://www.cisco.com /warp /public/732/Tech/netflow.

[18] V. Paxson. Bro: a system for detecting network intruders in real-time. In Computer Networks, volume 31, 1999.

[19] D. Plonka. Flowscan: A network traffic flow reporting and visualization tool. In USENIX LISA, Dec. 2000.

[20] M. Roesch. Snort - lightweight intrusion detection for networks. In USENIX LISA, 1999.

[21] M. Roughan, S. Sen, O. Spatscheck, and N. Duffield. Class-of-service mapping for QoS: A statistical signature-based approach to IP traffic classification. In $I M C$, Oct. 2004.

[22] Sampled NetFlow. http://www.cisco.com/ univercd/cc/td/ doc/product/software/ios120/ 120 newft/120limit/120s/120s11/12s_sanf.htm. 